Imperial/TP/94-95/20

\title{
Convergence of the Optimized Delta Expansion for the Connected Vacuum Amplitude: Anharmonic Oscillator
}

\author{
C. Arvanitis, H. F. Jones and C. S. Parker \\ Physics Department, Imperial College, \\ London SW7 2BZ, United Kingdom.
}

\begin{abstract}
The convergence of the linear $\delta$ expansion for the connected generating functional of the quantum anharmonic oscillator is proved. Using an order-dependent scaling for the variational parameter $\lambda$, we show that the expansion converges to the exact result with an error proportional to $\exp \left(-c N^{1 / 3}\right)$.
\end{abstract}

PACS Numbers : 11.15.Tk, 11.10Jj 


\section{Introduction}

The linear delta expansion (LDE) is an analytic approach to field theory which has been applied to a variety of problems (see for example Ref. [1]). The approach is non-perturbative in the sense that a power series expansion is made in a parameter $\delta$ artificially inserted into the action which interpolates between a soluble action $S_{0}$ and the action for the desired theory $S$. The action is written

$$
S_{\delta}=(1-\delta) S_{0}+\delta S
$$

where $S_{0}$ contains some dependence on a variational parameter $\lambda$. The generating functional for the theory may be evaluated as a power series in $\delta$, which of course is usually evaluated to finite order. When $\delta$ is set equal to one at the end of a truncated calculation of, say, a Green function $G$, some dependence on $\lambda$ remains, which is where the variational procedure makes its appearance. One such procedure is the principle of minimal sensitivity (PMS) [2] which requires $\lambda$ to be a stationary point of the truncated Green function $G_{N}$ :

$$
\frac{\partial G_{N}(\lambda)}{\partial \lambda}=0
$$

The PMS can provide both non-perturbative behaviour and convergence. It has been shown to ensure the convergence of the linear delta expansion for the vacuum generating functional for $\phi^{4}$ theories in both zero [3] and one [4] dimension. For the finite-temperature partition function $Z_{N}(\beta)$ at odd $N$ there is only one stationary point, a global maximum, so there is no ambiguity. Problems can, however, arise when there are multiple solutions to the PMS condition Eq. (2), as is the case [5] for the connected vacuum generating functional $W=\ln Z$ in zero dimensions. There, rather than applying the PMS directly to $W$, an order-dependent scaling for $\lambda$ was chosen which guarantees convergence for $W$. This was in fact the scaling resulting from the application of the PMS to the calculation of $Z$. In the present paper, we use a similar choice of scaling to provide a convergent series of approximants for the connected vacuum generating functional of the anharmonic oscillator.

The simplest choice of delta expansion used in Ref. [⿴囗口 (using as trial action a free action with variable mass) gives the finite-temperature partition function as a function of $z$, the complex extension of $\delta$ :

$$
Z(z)=\frac{1}{Z_{0}} \int_{x(0)=x(\beta)} D x \exp \left[-\int_{0}^{\beta} d \tau\left(\frac{1}{2} \dot{x}^{2}+\frac{1}{2}\left(m^{2}+2 g \lambda\right) x^{2}+z g\left(x^{4}-\lambda x^{2}\right)\right)\right]
$$

where $Z_{0}$ is the partition function for $g=0$. It is this system that we consider here. The scaling required for the PMS which also gives convergence was shown in Ref. \#4 to be $\lambda \propto N^{2 / 3}$. In the present paper, we use this scaling to prove the convergence of the $\delta$ expansion for $W=\ln Z$, provided that $m^{2}>0$. This scaling is not strictly PMS, simply a choice to give guaranteed convergence. 
In the following Sec. 2, we review the proof of convergence in the zero dimensional model. This serves as a detailed preview of the arguments used in the main sections of

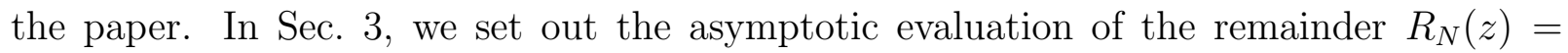
$Z(z)-Z_{N}(z)$ for the anharmonic oscillator. The analysis is closely related to that of

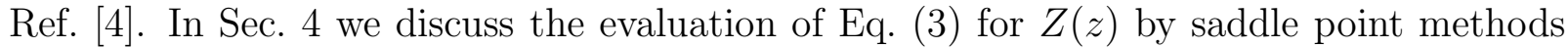
and the cancellations that govern the convergence of $W_{N}$. We compare our analysis with numerical calculations in Sec. 5, and summarize our results in the conclusions, Sec. 6.

\section{Illustration of the method}

The method of the present paper is similar in spirit to that of Ref. [5], which considers the zero dimensional analogue of the vacuum generating functional, namely:

$$
Z^{[0]}(z)=\int_{-\infty}^{\infty} d x \exp \left(-\left[\lambda(1-z) x^{2}+g z x^{4}\right]\right)
$$

An expansion of $Z^{[0]}$ in powers of $z$ was proved in Ref. [3] to converge to the correct value for $Z^{[0]}(1)$ with an error like $e^{-c N}$. The PMS condition guarantees convergence and requires $\lambda$ to scale as $\sqrt{N}$.

In Ref. [5] it was shown that the convergence of $W_{N}=\left.\left\{\ln Z_{N}(z)\right\}_{N}\right|_{z=1}$, where $\{f(z)\}_{N}$ denotes the Taylor expansion of $f(z)$ truncated at $O\left(z^{N}\right)$, is governed by the position of the roots of $Z_{N}(z)$. If any of these roots lies inside the circle $|z| \leq 1$, the expansion for $W_{N}(z)$ is not convergent, and in fact the position of the smallest modulus root determines the rate of convergence of the series for $W_{N}$. The difficulty in such a proof is that while it is a relatively simple matter to calculate the coefficients $c_{n}$ in the series $Z_{N}(z)=\Sigma c_{n} z^{n}$ (using simple integrals in zero dimensions or the methods of Caswell [G] and Killingbeck [7] in one dimension), finding the roots analytically is highly non-trivial.

In order to circumvent this difficulty, we write

$$
Z_{N}(z)=Z(z)-R_{N}(z)
$$

From this point of view, zeros of $Z_{N}(z)$ arise from a cancellation between $Z(z)$ and $R_{N}(z)$, each of which can be evaluated by asymptotic methods. In Ref. [5], the remainder $R_{N}(z)$ is bounded quite simply, while $Z(z)$ is estimated by stationary phase integration.

The saddle points of the exponent $S$ in Eq. (4) are $x_{0}=0$ and $x_{ \pm}= \pm \lambda(1-z) / 2 g z$. The mechanism identified in Ref. [0] for roots to lie inside the circle of mean radius $|z|=$ $\left|c_{0} / c_{N}\right|^{1 / N}$ was cancellation between the saddle-point contributions from $x_{0}$ and $x_{ \pm}$. This can only occur along the Stokes line $\Gamma$ defined by $\operatorname{Re} S\left(x_{ \pm}\right)=0$. However, part of $\Gamma$ lies inside $|z|=1$, and cancellation along this part of the curve would have been disastrous for the proof of convergence. Such cancellations were ruled out in Ref. [5] by explicitly constructing the stationary phase paths for different values of $z$. It was shown that for $|z|<1$ the correct path did not pass through $x_{ \pm}$, in contrast to the region $|z|>1$, where all three saddle points were traversed. 
Because this argument is difficult to generalize to a genuine path integral, we note here a simpler argument ruling out dominant contributions from $x_{ \pm}$in the region $\operatorname{Re} z<1$. In fact $Z^{[0]}(z)$ may be evaluated exactly as a modified Bessel function $K_{\frac{1}{4}}\left((\lambda(1-z))^{2} /\left(8 g^{2} z^{2}\right)\right)$, provided that Re $z<1$. The asymptotic behaviour of this function as $\lambda \rightarrow \infty$ has no exponential dependence on $N$ in this region. Thus saddle points potentially giving a greater contribution than the Gaussian saddle point $x_{0}$ are excluded. The argument immediately generalizes to the left half plane without the need for contour rotation. The analysis in the present paper is similar, though we are unable to escape the need for contour rotation.

In the region $\operatorname{Re} z>1$ all three saddle points can occur, with possible cancellations along the curve $\Gamma$. In this way it was shown that the smallest zero of $Z^{[0]}$ occurs at $z_{\min }=a+\sqrt{a^{2}-1}$ where $a=1+3 \pi i /(N \alpha)$, with $\alpha=1.3254 \ldots$. This tends to unity sufficiently slowly as $N$ increases to ensure that $W_{N}^{[0]}$ converges to $W^{[0]}$ with an error of the order of $\exp (-\sqrt{3 \pi N / \alpha})$.

\section{Convergence of the remainder $R_{N}(z)$}

The remainder $R_{N}=Z(z)-Z_{N}(z)$ can be written as

$$
\begin{aligned}
R_{N}(z)= & \frac{1}{Z_{0}} \int_{x(0)=x(\beta)} D x \exp \left[-\int_{0}^{\beta} d \tau\left(\frac{1}{2} \dot{x}^{2}+\frac{1}{2}\left(m^{2}+2 g \lambda\right) x^{2}+z g\left(x^{4}-\lambda x^{2}\right)\right)\right] \\
& \times\left[1-\Theta_{N}(y)\right]
\end{aligned}
$$

where $\Theta_{N}(y):=e^{-y}\left\{e^{y}\right\}_{N}$ and $y=-g z \int d \tau\left(x^{4}-\lambda x^{2}\right)$.

By integrating the identity for $\Theta_{N}$ given in Ref. [3]:

$$
\frac{d}{d y} \Theta_{N}=-\frac{y^{N} e^{-y}}{N !}
$$

we can write

$$
1-\Theta_{N}(y)=\int_{0}^{y} d y^{\prime} \frac{y^{\prime N} e^{-y^{\prime}}}{N !} .
$$

Since $z$ and hence $y$ is in general complex, we must make a change of variable dependent on the sign of the real part of $y$. For Re $y \geq 0$, we may write $y=|y| e^{i \theta}$ and change variables using $y^{\prime}=\omega e^{i \theta}$

$$
1-\Theta_{N}(y)=\int_{0}^{|y|} d \omega \frac{\omega^{N}}{N !} \exp \left(-\omega e^{i \theta}+i(N+1) \theta\right)
$$

For Re $y \leq 0$, we write $y=|y| e^{i(\theta+\pi)}(-\pi / 2 \leq \theta \leq \pi / 2)$ and use $y^{\prime}=\omega e^{i(\theta+\pi)}$ :

$$
1-\Theta_{N}(y)=\int_{0}^{|y|} d \omega \frac{\omega^{N}}{N !} \exp \left(-\omega e^{i \theta}+i(N+1)(\theta+\pi)\right)
$$


Applying these to the remainder Eq. (6) for $\operatorname{Re} z \geq 0$, we define weak and strong field regimes respectively by

$$
\begin{aligned}
\lambda \int_{0}^{\beta} d \tau x^{2} & \geq \int_{0}^{\beta} d \tau x^{4} \\
\lambda \int_{0}^{\beta} d \tau x^{2} & \leq \int_{0}^{\beta} d \tau x^{4} .
\end{aligned}
$$

We divide the remainder $R_{N}(z)$ into weak and strong field contributions $A_{N}$ and $B_{N}$, and find

$$
\begin{aligned}
\left|A_{N}(z)\right|= & \frac{1}{Z_{0}} \int_{A} D x \exp \left[-\int d \tau\left(\frac{1}{2} \dot{x}^{2}+\frac{1}{2}\left(m^{2}+2 g \lambda\right) x^{2}+g|z| \cos \theta\left(x^{4}-\lambda x^{2}\right)\right)\right] \\
& \times \int_{0}^{|y|} d \omega \frac{\omega^{N}}{N !} \exp (-\omega \cos \theta) \\
\left|B_{N}(z)\right|= & \frac{1}{Z_{0}} \int_{B} D x \exp \left[-\int d \tau\left(\frac{1}{2} \dot{x}^{2}+\frac{1}{2}\left(m^{2}+2 g \lambda\right) x^{2}+g|z| \cos \theta\left(x^{4}-\lambda x^{2}\right)\right)\right] \\
& \times \int_{0}^{|y|} d \omega \frac{\omega^{N}}{N !} \exp (\omega \cos \theta) .
\end{aligned}
$$

The two regimes are dealt with separately in the following subsections. For $\operatorname{Re} z<0$, we simply interchange the role of the weak and strong field contributions to $R_{N}(z)$. The calculations give a result similar to the zero dimensional case, namely that we may bound the remainder by

$$
\left|R_{N}(z)\right| \leq D|z|^{N+1} \exp \left(-c N^{2 / 3}\right) .
$$

The detailed derivation of Eq. (14) for $|z|=1$ and its reliance on the PMS scaling $\lambda=$ $\left(2 \gamma^{2} N^{2} / g\right)^{1 / 3}$ with $\gamma=0.186$ are given in Ref. $\llbracket$. For $|z| \neq 1$ we see that $R_{N}(z)$ is convergent for

$$
|z|<1+O\left(N^{-1 / 3}\right)
$$

\subsection{Strong fields}

This is the simpler regime to consider, as the analysis almost exactly mirrors that of Ref. [4]. Since the $\omega$ integrand in Eq. (13) is monotonically increasing, we may simply bound by the value at the upper limit:

$$
\begin{aligned}
\left|B_{N}(z)\right| \leq & \frac{1}{Z_{0} N !} \int_{B} D x \exp \left[-\int d \tau\left(\frac{1}{2} \dot{x}^{2}+\frac{1}{2}\left(m^{2}+2 g \lambda\right) x^{2}+g|z| \cos \theta\left(x^{4}-\lambda x^{2}\right)\right)\right] \\
& \times|y|^{N+1} \exp (|y| \cos \theta) .
\end{aligned}
$$

Now using $|y|=g|z| \int d \tau\left[x^{4}-\lambda x^{2}\right]$, we find that

$$
\begin{aligned}
\left|B_{N}(z)\right| \leq & \frac{|z|^{N+1}}{Z_{0} N !} \int_{B} D x \exp \left[-\int d \tau\left(\frac{1}{2} \dot{x}^{2}+\frac{1}{2}\left(m^{2}+2 g \lambda\right) x^{2}\right)\right] \\
& \times\left(g \int d \tau\left(x^{4}-\lambda x^{2}\right)\right)^{N+1}
\end{aligned}
$$


which is just $|z|^{N+1}$ times the $B_{N}$ given in Ref. [4]. Thus with no further analysis we are able to bound the strong field remainder by

$$
\left|B_{N}(z)\right| \leq|z|^{N+1} C(m, g) \beta N^{4 / 3} \exp \left(-N S_{B}(\gamma)\right)
$$

with $S_{B}$ and $\gamma$ as defined in Ref. [4].

\subsection{Weak fields}

The weak field contribution to the remainder is given by

$$
\begin{aligned}
\left|A_{N}(z)\right|= & \frac{1}{Z_{0}} \int_{A} D x \exp \left[-\int d \tau\left(\frac{1}{2} \dot{x}^{2}+\frac{1}{2}\left(m^{2}+2 g \lambda\right) x^{2}+g|z| \cos \theta\left(x^{4}-\lambda x^{2}\right)\right)\right] \\
& \times \int_{0}^{|y|} d \omega \frac{\omega^{N}}{N !} \exp (-\omega \cos \theta) .
\end{aligned}
$$

If we make a further change of variable $\omega=g|z| \sigma \int d \tau\left[\lambda x^{2}-x^{4}\right]$, the familiar factor $|z|^{N+1}$ already emerges:

$$
\begin{aligned}
\left|A_{N}(z)\right|= & \frac{|z|^{N+1}}{Z_{0} N !} \int_{0}^{1} d \sigma \int_{A} D x \exp \left[-\int d \tau\left(\frac{1}{2} \dot{x}^{2}+\frac{1}{2}\left(m^{2}+2 g \lambda\right) x^{2}\right)\right] \\
& \times \sigma^{N}\left(g \int d \tau\left(\lambda x^{2}-x^{4}\right)\right)^{N+1} \exp \left[g|z| \cos \theta(1-\sigma) \int d \tau\left(\lambda x^{2}-x^{4}\right)\right]
\end{aligned}
$$

For the allowed range of $\theta$, it is then clear that

$$
\begin{aligned}
\left|A_{N}(z)\right| \leq & \frac{|z|^{N+1}}{Z_{0} N !} \int_{0}^{1} d \sigma \int_{A} D x \exp \left[-\int d \tau\left(\frac{1}{2} \dot{x}^{2}+\frac{1}{2}\left(m^{2}+2 g \lambda\right) x^{2}\right)\right] \\
& \times \sigma^{N}\left(g \int d \tau\left(\lambda x^{2}-x^{4}\right)\right)^{N+1} \exp \left[g|z|(1-\sigma) \int d \tau\left(\lambda x^{2}-x^{4}\right)\right]
\end{aligned}
$$

We may then further bound $A_{N}(z)$ by removing the term $(1-\sigma) g z x^{4}$ from the last exponent in Eq. (21). We also use Stirling's approximation for the factorial. We follow Ref. [1] and allow the calculation to proceed for either sign of $m^{2}$ by writing:

$$
\begin{aligned}
\left|A_{N}\right|< & \frac{|z|^{N+1}}{\sqrt{2 \pi N} Z_{0}} \int_{0}^{1} d \sigma \int_{A} D x\left(g \int d \tau\left(\lambda x^{2}-x^{4}\right)\right) \exp \left[-\int_{0}^{\beta} d \tau\left(\frac{1}{2} \dot{x}^{2}+\frac{1}{2}\left|m^{2}\right| x^{2}\right)\right] \\
& \times \exp \left(-N S_{A}(x, \sigma, z)\right)
\end{aligned}
$$

where

$$
\begin{aligned}
S_{A}(x, \sigma, z)= & \int_{0}^{\beta} d \tau\left(\frac{x^{2}}{N}\left(g \lambda(1-|z|(1-\sigma))+m^{2} \theta\left(-m^{2}\right)\right)\right) \\
& -\ln \left(\frac{g \sigma}{N} \int_{0}^{\beta} d \tau\left(\lambda x^{2}-x^{4}\right)\right)-1,
\end{aligned}
$$


which for $z=1$ is equal to the $S_{A}$ defined in Ref. [4].

The calculation proceeds in exactly the same manner as in Ref. [4]], with the definition $\int_{0}^{\beta} x^{2} d \tau=\beta \lambda U$ and the use of the Cauchy-Schwarz identity to show that

$$
S_{A} \geq \tilde{\alpha} U-\ln (\alpha U(1-U))-1,
$$

with $\alpha$ and $\tilde{\alpha}$ the $z \neq 1$ extensions of those defined in Ref. [4]:

$$
\begin{aligned}
& \alpha=\frac{g \beta \lambda^{2} \sigma}{N} \\
& \tilde{\alpha}=\alpha\left(|z|+\frac{1-|z|}{\sigma}\right)+\frac{m^{2} \beta \lambda}{N} \theta\left(-m^{2}\right) .
\end{aligned}
$$

We minimize the bound on $S_{A}$ with respect to $U$ and take the minimum at

$$
U=\frac{1}{2}+\frac{1}{\tilde{\alpha}}\left[1-\sqrt{1+\frac{\tilde{\alpha}^{2}}{4}}\right],
$$

which gives a similar result to that of Ref. 四:

$$
S_{A} \geq F(\tilde{\alpha})-\ln \left(\frac{\alpha}{2}\right)
$$

where

$$
F(\tilde{\alpha})=\frac{\tilde{\alpha}}{2}-\sqrt{1+\frac{\tilde{\alpha}^{2}}{4}}+\ln \left(1+\sqrt{1+\frac{\tilde{\alpha}^{2}}{4}}\right) .
$$

It is true for the present definition of $\tilde{\alpha}$ that for $\lambda \propto N^{2 / 3}$ and large $N$,

$$
\frac{\partial}{\partial \alpha}\left[F(\tilde{\alpha})-\ln \left(\frac{\alpha}{2}\right)\right]<0,
$$

so that the integral over $\sigma$ in Eq. (22) is dominated by its value at the upper limit. Thus:

$$
\begin{aligned}
\left|A_{N}\right|< & \frac{|z|^{N+1}}{\sqrt{2 \pi N} Z_{0}} \int_{A} D x\left(g \int d \tau\left[\lambda x^{2}-x^{4}\right]\right) \exp \left[-\int_{0}^{\beta} d \tau\left[\frac{1}{2} \dot{x}^{2}+\frac{1}{2}|m|^{2} x^{2}\right]\right] \\
& \times \exp \left[-N\left(F\left(\tilde{\alpha}_{0}\right)-\ln \left(\frac{1}{2} \alpha_{0}\right)\right)\right]
\end{aligned}
$$

with the subscript 0 on $\alpha$ and $\tilde{\alpha}$ denoting $\sigma=1$, giving

$$
\begin{aligned}
& \alpha_{0}=\frac{g \beta \lambda^{2}}{N} \\
& \tilde{\alpha}_{0}=\alpha_{0}+\frac{m^{2} \beta \lambda}{N} \theta\left(-m^{2}\right) .
\end{aligned}
$$


Now if we use the relation: $g \int_{0}^{\beta} d \tau\left[\lambda x^{2}-x^{4}\right] \leq g \beta \lambda^{2} / 4$ and the fact that the functional integral in the numerator of Eq. (30) is bounded above by that in the denominator, we obtain

$$
\left|A_{N}\right| \leq \frac{g \beta \lambda^{2}|z|^{N+1}}{4 \sqrt{2 \pi N}} \exp \left[-N\left(F\left(\tilde{\alpha}_{0}\right)-\ln \left(\frac{\alpha_{0}}{2}\right)\right)\right]
$$

Using the scaling $\lambda \propto N^{2 / 3}$ we have $\tilde{\alpha}_{0} \approx \alpha_{0}\left[1+O\left(N^{-2 / 3}\right)\right]$. For large $N, \alpha \propto N^{1 / 3}$ and we may finally write

$$
\left|A_{N}(z)\right| \leq|z|^{N+1} N^{5 / 6} \exp \left[-c N^{2 / 3}\right]
$$

\section{Saddle point expansion of $Z(z)$}

The partition function may be written as

$$
Z(z)=\frac{1}{Z_{0}} \int_{x(0)=x(\beta)} D x \exp (-S[x])
$$

where

$$
S[x]:=\int_{0}^{\beta} d \tau\left(\frac{1}{2} \dot{x}^{2}+\frac{1}{2} M^{2} x^{2}+g z x^{4}\right)
$$

and $M^{2}=m^{2}+2 g \lambda(1-z)$, which is large for $\lambda \propto N^{2 / 3}$ and $z \neq 1$.

Performing a saddle point expansion of the path integral requires finding the solutions of the Euclidean equation of motion

$$
\ddot{x}=M^{2} x+4 g z x^{3},
$$

with $x(0)=x(\beta)$, which has the static solutions:

$$
\begin{aligned}
& x=x_{0}=0 \\
& x=x_{ \pm}= \pm \sqrt{-\frac{M^{2}}{4 g z}}
\end{aligned}
$$

There is also a non-static instanton solution. This is discussed by Zinn-Justin [8] in the case of negative $g$ and real $M^{2}>0$. This class of solution also arises in the strong coupling contribution to the remainder in Ref. [1], the modified action giving an equation of motion equivalent to negative coupling. This solution is

$$
x_{I}(\tau)=\sqrt{\frac{-M^{2}}{2 g z}} \frac{1}{\cosh \left(M\left(\tau-\tau_{0}\right)\right)},
$$

where $\tau_{0}$ must strictly be taken as $\tau_{0}=\frac{1}{2} \beta$ to satisfy the periodic boundary conditions. We note that this solution takes the form $1 / \cos \left(M\left(\tau-\tau_{0}\right)\right)$ for real $z$, such that $z>$ $1+m^{2} /(2 g \lambda)$. For general complex $z$ the solution is an hybrid. 
A more general class of solutions is discussed by Richard and Rouet [9] for the double well oscillator. The action of Eq. (35) may be rescaled to the form of Ref. [9] which is:

$$
S_{R R}[x]=\int_{-T}^{T} d t\left[\frac{1}{2} \dot{x}^{2}+\frac{1}{2}\left(x^{2}-1\right)^{2}\right]
$$

The solutions to the modified equation of motion take the form

$$
x_{R R}(t)=a\left(1+\frac{a^{2}-1}{\mathcal{P}\left(t+u \mid \omega, \omega^{\prime}\right)+\frac{1}{6}-\frac{1}{2} a^{2}}\right)
$$

where $a$ is an integration constant and $\mathcal{P}\left(t+u \mid \omega, \omega^{\prime}\right)$ is a Weierstrass elliptic function [10 whose periods $\omega, \omega^{\prime}$ are determined by the boundary conditions and form of the elliptic integral inverted by Eq. (41). The cosine instanton solution discussed above is equivalent to Eq. (41) with $\omega^{\prime} / \omega \rightarrow i \infty$.

The classical action for all the saddle points at large $M$ may be evaluated simply:

$$
\begin{aligned}
S\left[x_{0}\right] & =0 \\
S\left[x_{ \pm}\right] & =-\frac{M^{4} \beta}{16 g z} \\
S\left[x_{I}\right] & =-\frac{M^{3}}{3 g z} \\
S\left[x_{R R}\right] & =S\left[x_{ \pm}\right]+k S\left[x_{I}\right],
\end{aligned}
$$

where $k$ is a positive integer, except that in the case $\omega^{\prime} / \omega \rightarrow i \infty$, cancellations occur in the action of Richard and Rouet to give $S\left[x_{R R}\right]=S\left[x_{I}\right]$.

Having evaluated the classical actions, we must evaluate the regions of the $z$ plane in which the various saddle points are dominant. These regions are illustrated in Fig. 1 for the numerical values $m=1, g=1 / 2, \beta=2$ and $\lambda=11.619(N=75)$. The solid curve $\Gamma$ is the curve $\operatorname{Re} S\left[x_{ \pm}\right]=0$ and the dotted curve $\Gamma_{I}$ is the curve $\operatorname{Re} S\left[x_{I}\right]=0$, while the dashed vertical line marks $\operatorname{Re} z=1+m^{2} / 2 g \lambda$. All three meet at the point $z=1+m^{2} / 2 g \lambda$.

Inside $\Gamma_{I}$, the Richard and Rouet saddle points are dominant. Inside the loop of $\Gamma$, the saddle point $x_{ \pm}$dominates over $x_{0}$, though this is unimportant given the overall dominance of $S\left[x_{R R}\right]$. Outside $\Gamma_{I}$ the zero saddle point becomes dominant over all its competitors. This remains the case up to the boundary $\Gamma$ to region $\mathrm{C}$, in which $x_{ \pm}$is dominant. Neither $x_{I}$ nor $x_{R R}$ can dominate the integral in this region.

It would then appear that instantons dominate for small $|z|, x_{0}$ is dominant for intermediate $|z|$, and for large $|z|$ the saddle points $x_{ \pm}$are the important ones. However, as we learnt from the zero-dimensional case, it is important to check whether apparently dominant saddle points do in fact contribute. In zero dimensions Ref. [5] it was possible to trace out the stationary-phase paths and to see that these did not pass through the non-zero saddle points when $|z|<1$. For a path integral, such a procedure is extremely ill defined. However, we are able to use instead an analogue of the Bessel function analysis given above for the zero dimensional case by realizing that for $\operatorname{Re} z<1+m^{2} /(2 g \lambda)$ the 
action is generically a real single well, the complex part of the mass term simply adding a phase. Provided then that $\operatorname{Re}\left(g z x^{4}\right)>0$ the partition function is bounded above by that for the pure harmonic oscillator. The harmonic oscillator partition function is given by

$$
\begin{aligned}
\left|Z_{0}\right| & =\int D x \exp \left[-\int_{0}^{\beta} d \tau\left(\frac{1}{2} \dot{x}^{2}+\frac{1}{2} \operatorname{Re}\left(M^{2}\right) x^{2}\right)\right] \\
& =C\left(\operatorname{det}\left(\partial_{t}^{2}+\operatorname{Re} M^{2}\right)\right)^{-\frac{1}{2}}
\end{aligned}
$$

where $C$ is a normalization constant. The Gaussian integration about the saddle point $x=0$ in Eq. (34) gives:

$$
|Z|=C\left|\operatorname{det}\left(\partial_{t}^{2}+M^{2}\right)\right|^{-\frac{1}{2}} .
$$

We are thus able to state that in the region $0<\operatorname{Re} z<1+m^{2} /(2 g \lambda)$ there is in fact no exponentially increasing contribution from saddle points with $\operatorname{Re} S[x]<0$. The zero saddle point is the only one which can contribute. From the form of Eq. (14), it is then clear that no zeros of $Z_{N}(z)$ are possible for $|z|<1+O\left(N^{-1 / 3}\right)$ in the region $0<\operatorname{Re} z<1+m^{2} /(2 g \lambda)$. In fact the zeros will be expected to occur on a ring of radius $1+O\left(N^{-1 / 3}\right)$.

The left half of the $z$ plane may be included by rotating the contour of integration to make $\operatorname{Re}\left(z x^{4}\right)>0$ while keeping both $\operatorname{Re}\left(\dot{x}^{2}\right)>0$ and $\operatorname{Re}\left(M^{2} x^{2}\right)>0$. This procedure is similar to that discussed in Ref. [8]. In the upper half plane, we rotate the contour by an angle $\phi=(\pi / 2-\theta) / 4$, where $\theta=\arg z$. In the lower half plane, we simply rotate the contour by an equivalent amount, but in the opposite sense.

In the region $\operatorname{Re} z>1+m^{2} /(2 g \lambda)$, we need only consider the stationary saddle points $x_{0}$ and $x_{ \pm}$. In the region $\mathrm{B}, x_{0}$ dominates and the requirements for cancellation are the same as those discussed above. No zeros affecting the rate of convergence of $W_{N}$ can arise in this region for $m^{2}>0$, and we expect the ring of zeros to continue smoothly into this region. For the non-Borel summable case $m^{2}<0$, this proof breaks down, since zeros of $Z_{N}(z)$ could now occur for $|z| \leq 1$.

In region $\mathrm{C}$, where $x_{ \pm}$is the dominant saddle point, $Z(z)$ contains exponential dependence on $N$, allowing cancellation with $R_{N}(z)$, which grows with $|z|$ for $|z|>1+O\left(N^{1 / 3}\right)$. The zeros in region $\mathrm{C}$ will thus tend to be further out than those in $\mathrm{B}$.

An exception to this is the region close to $\Gamma$, the Stokes line where $\operatorname{Re} S\left[x_{ \pm}\right]=0$. There cancellations can occur between the two saddle-point contributions from $x_{0}$ and $x_{ \pm}$, giving a smaller effective exponent for $Z(z)$, and the possibility of roots of smaller modulus. However, since this branch of $\Gamma$ lies to the right of Re $z=1+m^{2} /(2 g \lambda)$, such zeros again do not affect the convergence of $W_{N}$ for $m^{2}>0$. Having established that all the roots satisfy $|z-1|>m^{2} / 2 g \lambda=O\left(1 / N^{2 / 3}\right)$, the analysis of Ref. [5] shows us that $W_{N}$ tends to the correct value $W$ with an error

$$
\begin{aligned}
\mathcal{R}_{N} & :=W-W_{N} \\
& \propto \exp \left(-c N^{1 / 3}\right)
\end{aligned}
$$




\section{Comparison with numerical results}

The above analysis of the position of the roots of $Z_{N}(z)$ can be checked numerically. Figs. 2, 3 and 4 show the roots of $Z_{N}(z)$ for $\beta=2, m=1, g=1 / 2$ and $N=25,45$ and 75 respectively. In each case, $\lambda$ is chosen as the PMS value for $Z_{N}(z)$. In the previous section, it was noted that the smallest root occurs where $\operatorname{Re} S\left[x_{ \pm}\right]=0$. In order to make the comparison, we have plotted the curve $\Gamma$ on each graph.

In Fig. 2 we see that at order 25, the roots lie to a good approximation on a ring, the radius of which is determined by the ratio of the first and last coefficients in the $\delta$ series. Fig. 3, for order 45, shows that two pairs of roots have broken away from this ring, one pair moving out and one pair moving in. The inner pair of roots have the smallest modulus, and lie sufficiently close to $\Gamma$ to indicate that the mechanism outlined above is indeed operative. At order 75 in Fig. 4, two pairs of roots are tracking in along the curve $\Gamma$. This behaviour very closely follows that described in Ref. [5] for the zero dimensional case.

We have checked the convergence of the expansion for $W$ numerically. Taking $\lambda_{N}$ as the unique PMS value for $Z_{N}$ we obtain Table 1 , showing convergence to 15 significant figures by $N=85$ for both $\beta=2$ and $\beta=5$. The convergence is not monotonic, as was the case for $Z_{N}$; rather it oscillates around the exact value with exponentially decreasing amplitude.

Notwithstanding the problem of multiple PMS points for $W_{N}(\lambda)$ itself, it is interesting to explore the dependence on $\lambda$ of $W_{N}$. For the values of $N$ we have considered there seems to be an initial, extremely broad maximum, which gives a very accurate estimate of $W$, followed by a series of secondary maxima and minima of progressively decreasing accuracy. For $N=45$ and $\beta=2$ the value at this first maximum is -1.353868180362611 , differing from the exact value only in the 16th. significant figure. A similar situation occurs for $\beta=5$.

\begin{tabular}{ccc}
$N$ & $W_{N}(\beta=2)$ & $W_{N}(\beta=5)$ \\
\hline 17 & -1.3538682048367371 & -3.4805935670797027 \\
25 & -1.3538681804666174 & -3.4805880725830912 \\
35 & -1.3538681803659796 & -3.4805878403631746 \\
45 & -1.3538681803626513 & -3.4805878324251261 \\
55 & -1.3538681803625918 & -3.4805878320234629 \\
65 & -1.3538681803626148 & -3.4805878319968700 \\
75 & -1.3538681803626174 & -3.4805878319947731 \\
85 & -1.3538681803626168 & -3.4805878319945885 \\
\hline Exact value & -1.3538681803626170 & -3.4805878319945603
\end{tabular}

Table 1: Results showing convergence of $\delta$ expansion for $W_{N}$ 


\section{Conclusions}

In this paper, we have proved the convergence of the optimized $\delta$ expansion for $W=\ln Z$, where $Z$ is the finite-temperature partition function of the anharmonic oscillator. With the variational parameter $\lambda$ chosen to scale with the order $N$ of the expansion as

$$
\lambda=\left(2 \gamma^{2} N^{2} / g\right)^{1 / 3}
$$

we found that

$$
W-W_{N} \propto \exp \left(-c N^{1 / 3}\right) .
$$

Apart from the fact that in a field theoretic context it is generally the connected Green functions which are the relevant quantities, the original motivation [5] for looking at $W$ was the non-uniform convergence with $\beta$ of the delta expansion for $Z_{N}$, in particular in the limit $\beta \rightarrow \infty$. Since this non-uniformity corresponds to the limit of large spacetime volume, it seemed plausible that the difficulty would be much less severe for $W$, the generating function of connected diagrams, which should depend only linearly on the (large) volume cut-off. However, the present proof of convergence for $W_{N}$ does not address this problem, since it relies as an intermediate step on the convergence of the sequence $Z_{N}$. It remains an open question whether the PMS criterion applied directly to $W_{N}$ itself, in spite of the problems of multiple stationary points, can give rise to uniform convergence as $\beta \rightarrow \infty$.

In the meantime a paper by Guida, Konishi and Suzuki [11] has appeared, which, using a completely different approach involving a dispersion relation in $g$, has proved convergence of the $\delta$ expansion for the individual energy levels of the anharmonic oscillator for a variety of scaling exponents $\eta$ in $\lambda_{N} \propto N^{\eta}$. The PMS choice we have used here, with $\eta=2 / 3$, lies at the edge of their range $2 / 3 \leq \eta<1$. It is possible that their method could be extended to a proof of convergence for $W$, or the free energy $F=W / \beta$.

The proof of Ref. 11] is restricted to $m^{2}>0$, as is the case here, even though it was shown in Ref. [4] that for $Z$ the sequence of approximants $Z_{N}$ converges for either sign of $m^{2}$. As a general principle the field theory about which we expand should capture as closely as possible the essential features of the system under investigation [12]. For the double-well oscillator it may well be that to obtain a convergent expansion for the connected Green functions a more sophisticated trial action is needed than the one used here and in Ref. [11], namely a free action with positive $m^{2}$.

The extension of the method to higher dimensions should be possible, since only saddle point techniques are used. In higher dimensional field theories we must, however, take account of the interplay between the $\delta$ expansion and the renormalization procedure. Such a scheme has been successfully applied to the Gross-Neveu model in the large $N$ limit using the $\delta$ expansion [13] and a related scheme [14 and $\phi^{4}$ theory in four dimensions in the Gaussian approximation [15]. 


\section{Acknowledgements}

C. A. is grateful to the theory group at Imperial College for their kind hospitality and acknowledges financial support from ECC grant no. ERBCHBICT941235.

\section{References}

[1] H. F. Jones in Proceedings of LP-HEP 91, p. 111 (World Scientific 1992).

[2] P. M. Stevenson: Phys. Rev. D23, 2916 (1981).

[3] I. R. C. Buckley, A. Duncan and H. F. Jones: Phys. Rev. D47, 2554 (1993).

[4] A. Duncan and H. F. Jones: Phys. Rev. D47, 2560 (1993).

[5] C. M. Bender, A. Duncan and H. F. Jones: Phys. Rev. D49, 4219 (1994).

[6] W. E. Caswell, Ann. Phys. (N.Y.), 123, 153 (1979).

[7] J. Killingbeck, J. Phys. A14, 1005 (1981).

[8] J. Zinn-Justin: Quantum Field Theory and Critical Phenomena (Clarendon Press, Oxford, 1993).

[9] J. L. Richard and A. Rouet: Nucl. Phys. B183, 251 (1981); Nucl. Phys. B185, 61 (1981).

[10] P. F. Byrd and M. D. Friedman: Handbook of Elliptic Integrals for Engineers and Scientists (Springer-Verlag, Berlin, 1971).

[11] R. Guida, K. Konishi and H. Suzuki: Genova Preprint GEF - Th-7/1994, hep-th9407027.

[12] F. Dyson: Phys. Rev. 85, 631 (1952).

[13] H. F. Jones and M. Moshe: Phys. Lett. B234, 492 (1990).

[14] C. Arvanitis, F. Geniet and A. Neveu (unpublished).

[15] I. Stancu and P.M.Stevenson: Phys. Rev. D42, 2710 (1990). 


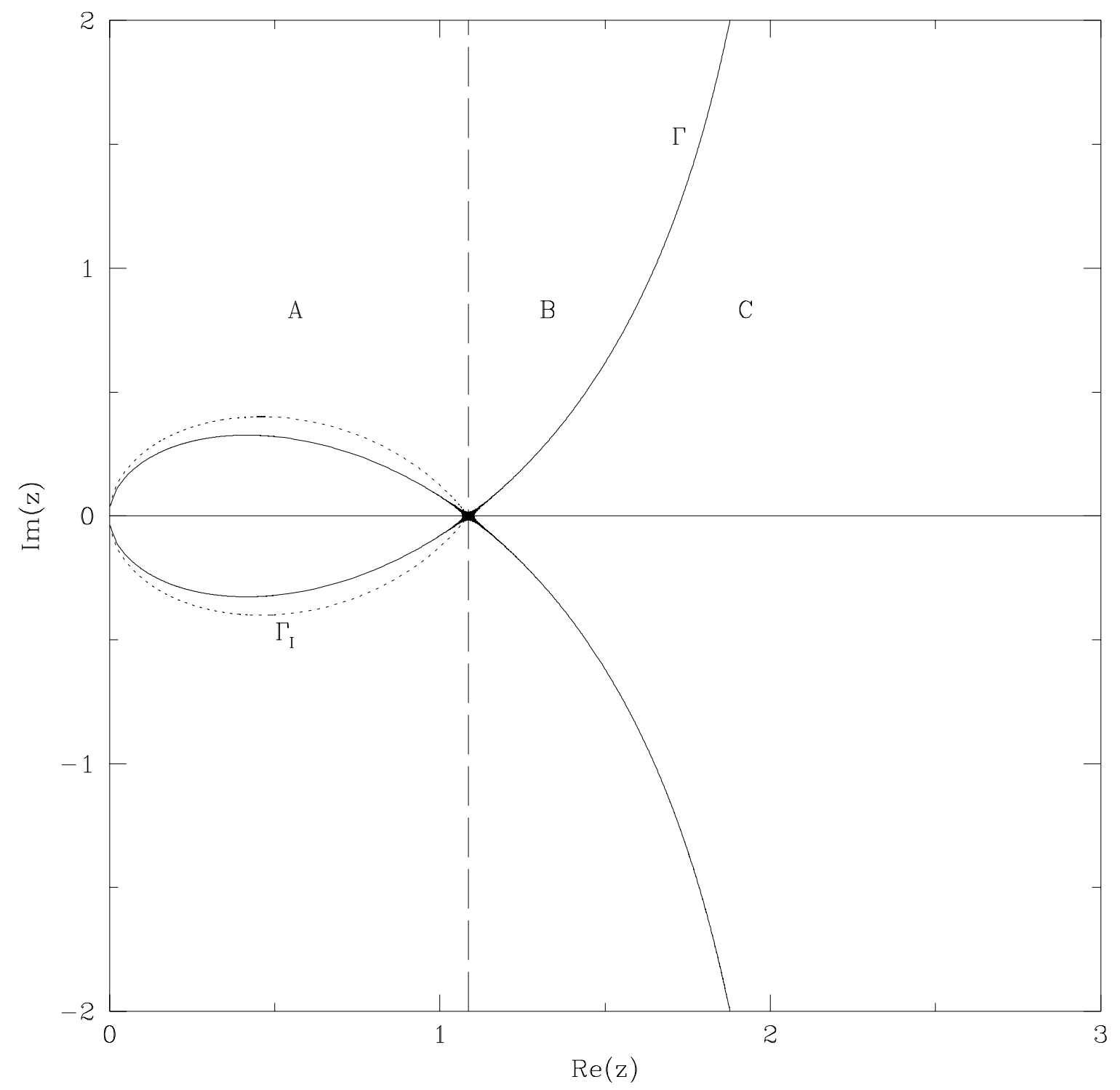

Figure 1: Plot of the $z$ plane showing the regions in which the possible saddle points are dominant. 


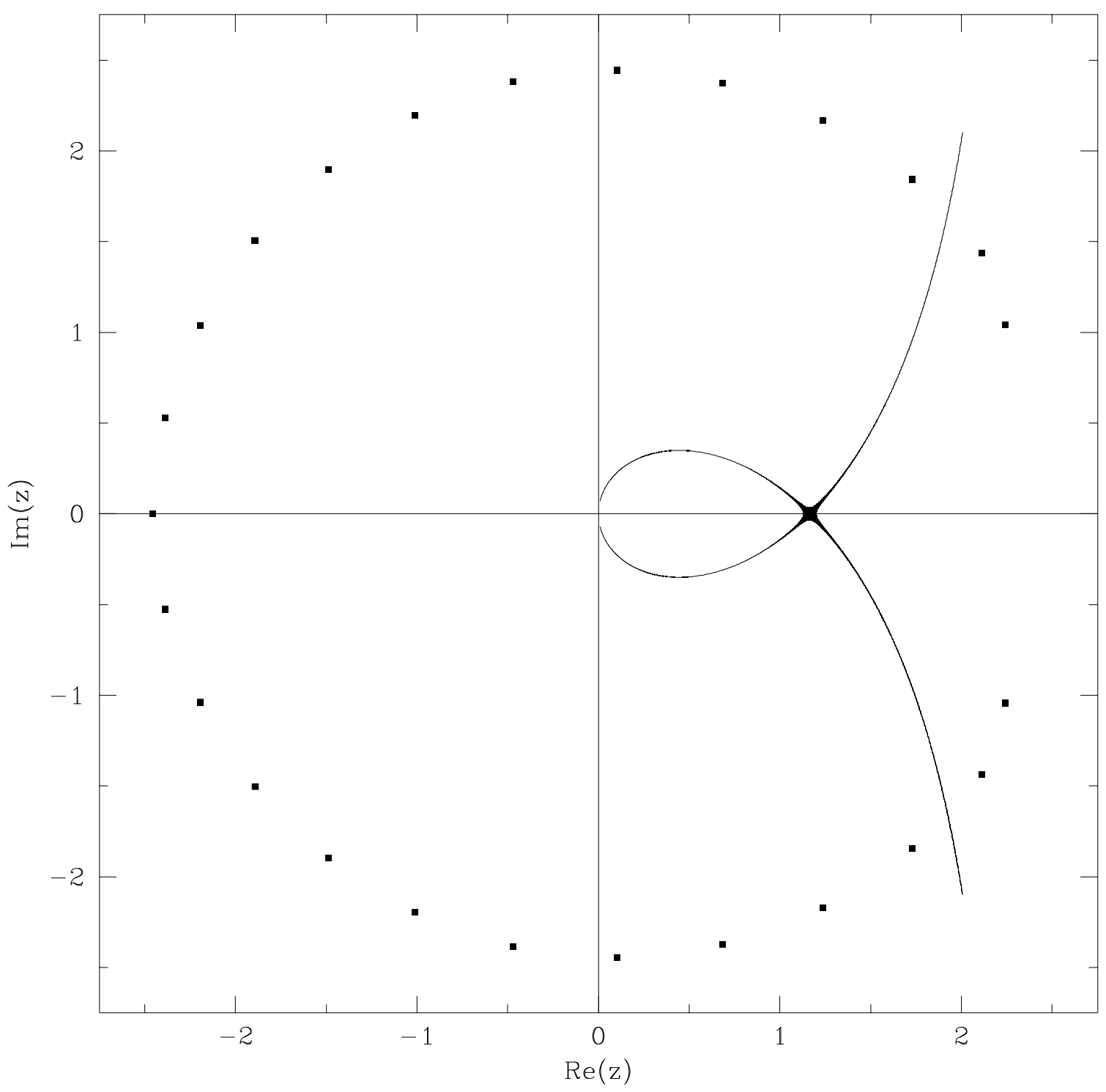

Figure 2: Numerical plot of roots of $Z_{N}(z)=0$ for $\beta=2, N=25$ and the curve $\Gamma$. 


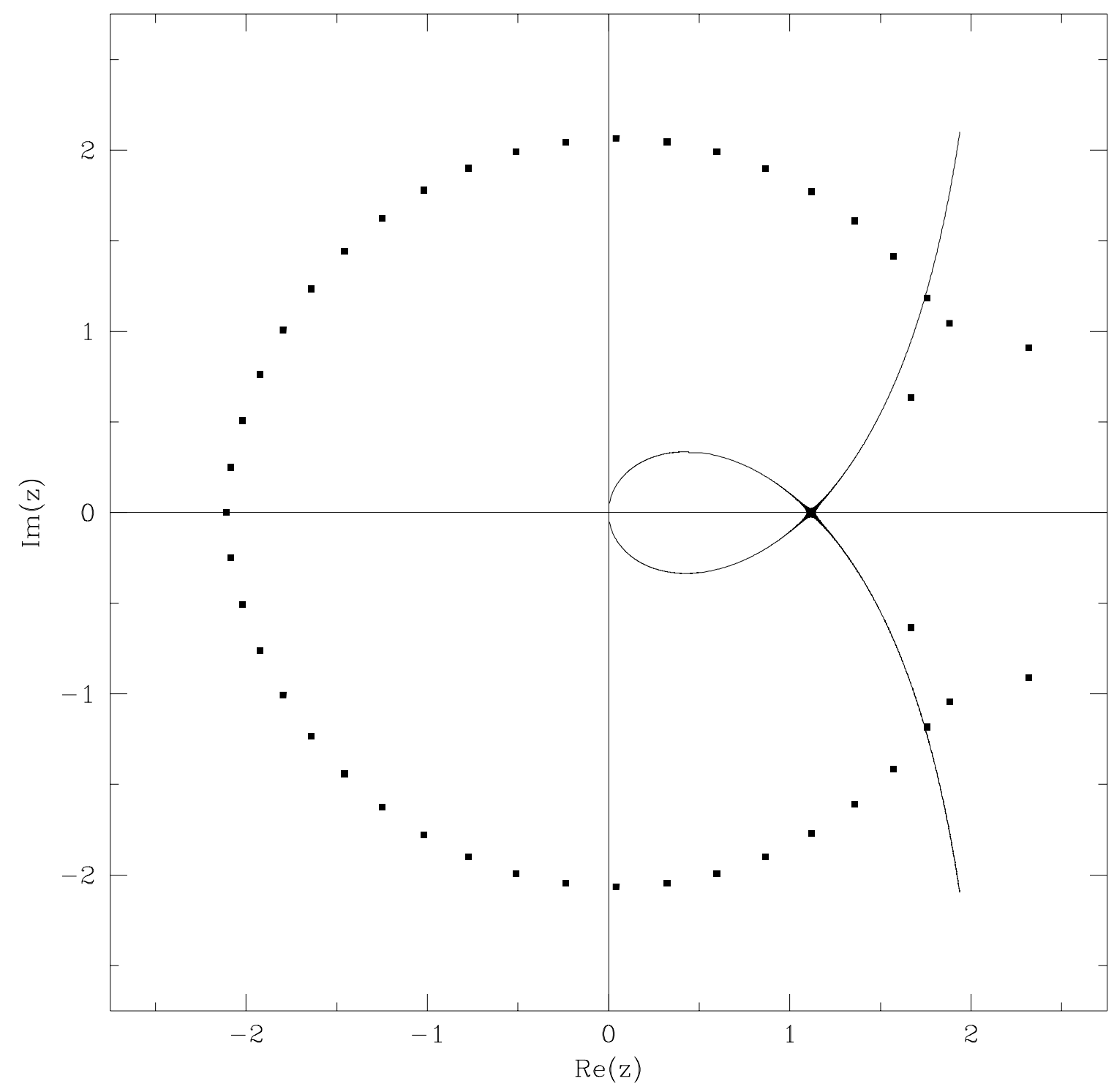

Figure 3: As Figure 2, for $N=45$. 


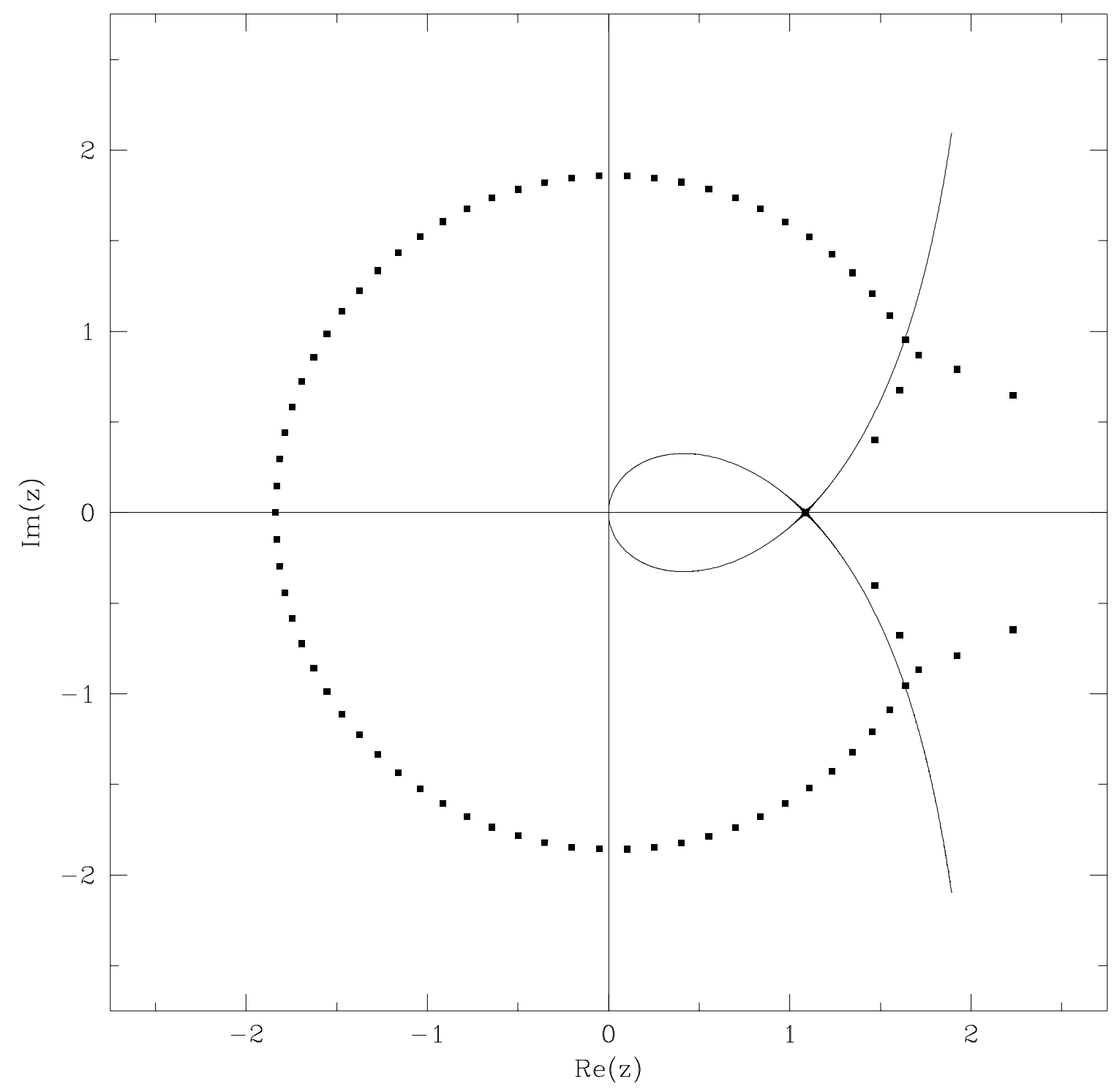

Figure 4: As Figure 2, for $N=75$. 